\title{
Passage of a vehicle over a standard obstacle
}

\author{
Darina Stachová1,** \\ ${ }^{1}$ University of Žilina, Faculty of Security Engineering, Department of Technical Sciences and \\ Informatics, Univerzitná 8215/1, 01026 Žilina, Slovak Republic
}

\begin{abstract}
Traffic safety depends on vehicle quality, traffic density and road conditions. Motion of a vehicle on a road or bridge body is therefore interesting from many points of view for various experts. We draw our attention to the unevenness of the road, specifically to the road profile with the so-called standard obstacle. In the article we present several alternatives for the mathematical model of such a road and also the mathematical model of the trajectory of the vehicle while traversing this standard obstacle. The shape of the obstacle meets the requirement of continuous one-point contact of the tire with the road; it does not contain singular points, and thus provides conditions for safer driving, especially when transporting persons in critical condition, or shocks-sensitive or hazardous materials.
\end{abstract}

\section{Introduction}

Every transportation company is interested in delivering undamaged goods safely to the destination. Of particular interest in this context it is the transport of chemically unstable substances and objects sensitive to shocks and impacts, e.g. gases, liquids, live animals, humans. Motion of the vehicle on a road or bridge body is therefore interesting from many points of view. It is of interest to the designers of vehicles themselves, to the designers of the road, to the engineers investigating the dynamical effects caused by the movement of vehicles [1] and many others. Road safety therefore requires limiting the maximum speed and, in special road sections, forced (deliberate) deceleration of the vehicles. For this purpose, artificial obstacles are placed on the road surface. These deliberately placed obstacles are called speed breakers. That's why road designers pay attention to the longitudinal profile of the road. In our paper we present a mathematical description of the standard shape of a speed breaker, the so-called standard obstacle (Fig. 1) and a description of the trajectory of the vehicle while passing through this obstacle.

\footnotetext{
*Corresponding author: darina.stachova@uniza.sk
} 


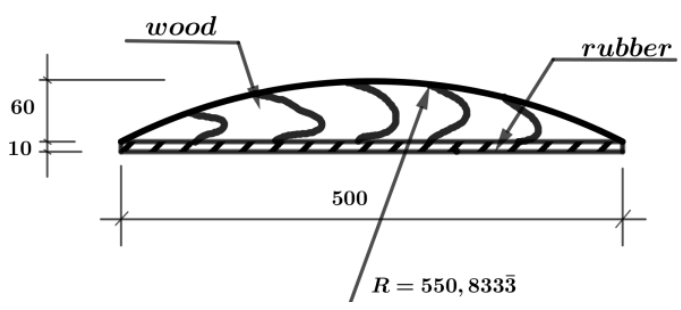

Fig. 1. Model of standard obstacle type.

\section{Simplified speed breaker type}

Deceleration thresholds - speed breakers are used on roads for the purpose of traffic calming according to TP 15/2005 SSC Principles of designing traffic calming elements on sections of roads in municipalities and towns. Prefabricated deceleration thresholds are available from parts manufactured in two sizes of $430 \mathrm{~mm} \times 500 \mathrm{~mm}$ or $430 \mathrm{~mm} \times 215 \mathrm{~mm}$. These obstacles are in the form of a cylindrical section with a height of $60 \mathrm{~mm}$ and a chord length of $500 \mathrm{~mm}$ [1]. It is made of two materials - wood and a flexible rubber pad. A simplified 2D model of the speed breaker is sufficient for the road designer to model the traffic situation. First, we describe the speed breaker made from a wood material. Its shape is derived from a circular arc.

The shape of the trajectory of a moving vehicle is determined by the shape of the road and its unevenness. The function describing such a roadway is continuous and composed of three different parts. The letter h denotes the height of the speed breaker, $l$ its width, $r$ the radius of the rolling wheel of the vehicle and $R$ the radius of the circular arc of the speed breaker. $R$ is dependent on given parameters 1 and $\mathrm{h}$ and the following holds: $R=\frac{4 h^{2}+l^{2}}{8 h}$. Let us denote $\alpha=\sqrt{2 h(r+R)-h^{2}}$.

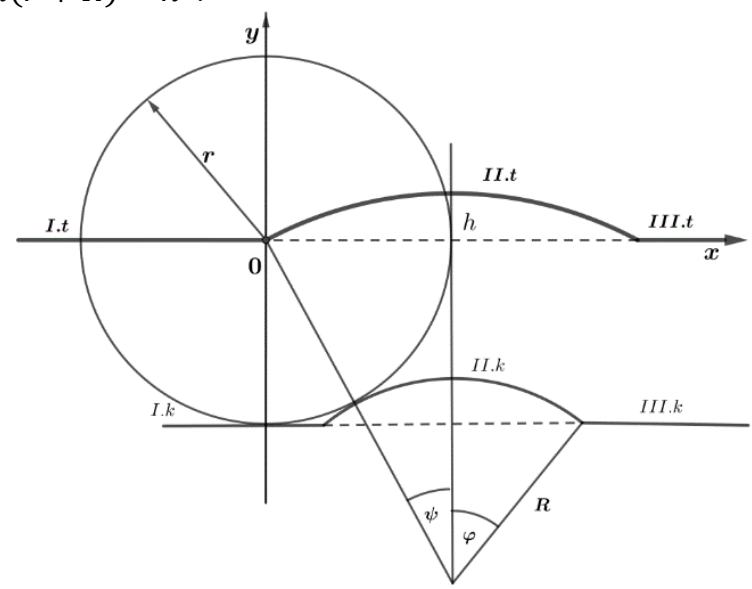

Fig. 2. Simplified speed breaker type.

The trajectory of a moving vehicle on the road with the speed breaker shown in Fig. 2 can be parametrically expressed as follows:

$$
\begin{aligned}
& \text { I.t a III.t: } x=(R+r-h) \tan t+\alpha \\
& y=0 \\
& \text { II.t: } \quad x=(R+r) \sin t+\alpha \\
& y=(R+r) \cos t+h-R-r \quad \text { where } t \in[-\psi ; \psi]
\end{aligned}
$$




\section{Standard obstacle}

The normal type of speed breaker is related to the one above. It is constructed from a circular arc that is shifted in the direction of $y$ axis by a vector $\vec{u}$. Parameters marked in the first type of retarder are identically identified for the second type.

Let us denote $\beta=\sqrt{2(R+r)(h+u)-(h+u)^{2}}$. The model of the road shape with standard obstacle has parametric equations:

$$
\begin{array}{lll}
\text { I. } k \text { and III. } k: & x=(R-h-u) \tan t+\beta & \\
& y=-r & \text { where } t \in\left[-\frac{\pi}{2} ;-\varphi\right. \\
\text { II. } k: & x=R \sin t+\beta x & \\
& y=r \cos t+h+u-R-r & \text { where } t \in[-\psi ; \psi]
\end{array}
$$

From the model of the road we derive the trajectory model of the vehicle moving along it. Its parametric equations are as follows:

$$
\begin{aligned}
& \text { I. } t \text { and III. } t: \quad x=[(R+r)-(h+u)] \tan t+\beta \\
& y=0 \\
& \text { II. } t: \quad x=(R+r) \sin t+\beta \\
& y=(R+r) \cos t+h+u-R-r \quad \text { where } t \in[-\vartheta ; \vartheta]
\end{aligned}
$$

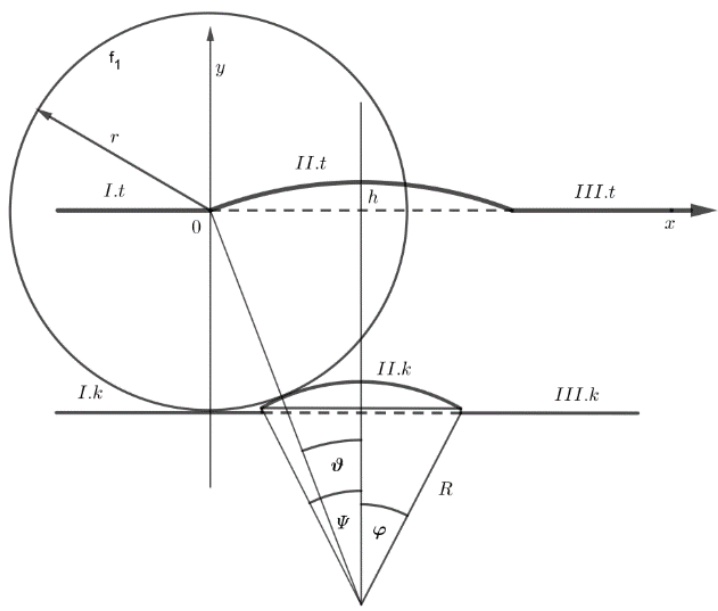

Fig. 3. Standard speed breaker type.

In both of the above types, we have neglected the deformations of the trajectory that are caused by the deformation of the tire when it encounters the speed breaker. Furthermore, we considered that there was a one-point contact between the vehicle and the road at all times [2]. Obviously, the vehicle can only follow the road surface if it does not exceed the speed limit. The height of the speed breaker $h$ depends on the speed to which we want to decelerate the vehicle. If the vehicle moves at a speed higher than that, it will lose contact with the road surface. The relationship between the desired speed and the height of the speed breaker is illustrated in Table 1.

Table 1. Speed dependence on obstacle dimensions.

\begin{tabular}{|l|l|}
\hline Desired speed $v$ & Speed breaker height $h$ \\
\hline $10 \mathrm{~km} / \mathrm{h}$ & $60 \mathrm{~mm}$ \\
\hline $20 \mathrm{~km} / \mathrm{h}$ & $50 \mathrm{~mm}$ \\
\hline $50 \mathrm{~km} / \mathrm{h}$ & $30 \mathrm{~mm}$ \\
\hline
\end{tabular}


It is for these reasons that the vehicle is forced to reduce its speed before encountering the speed breaker. These characteristics of the mathematical model of the speed breaker reflect the mathematical characteristics of the functions that describe its shape and the trajectory of the moving vehicle. Both have singular points, both of which are concave. The occurrence of singular points on a mathematical curve corresponds to the existence of points of multipoint contact between the vehicle tire and the ground. As a result, the vehicle does not copy the shape of the road as it moves. This reduces the safety of the movement and the safety of transported persons or material. For this reason in the next section we propose to model the optimal shape of the speed breaker.

\section{Optimized Type}

The optimized shape of the speed breaker requires that there be no singular points on the road but also on the trajectory of the vehicle, i.e., that its parts form smooth curves in the mathematical sense of the word. Since $R>h$, it is necessary to limit $l>761.3 \mathrm{~mm}$. We constructed the curve from five segments that consist of circular arcs and half lines touching each other at the connection points. We final curve is composed in such a way that the derivative at the endpoints is zero (Fig. 4).

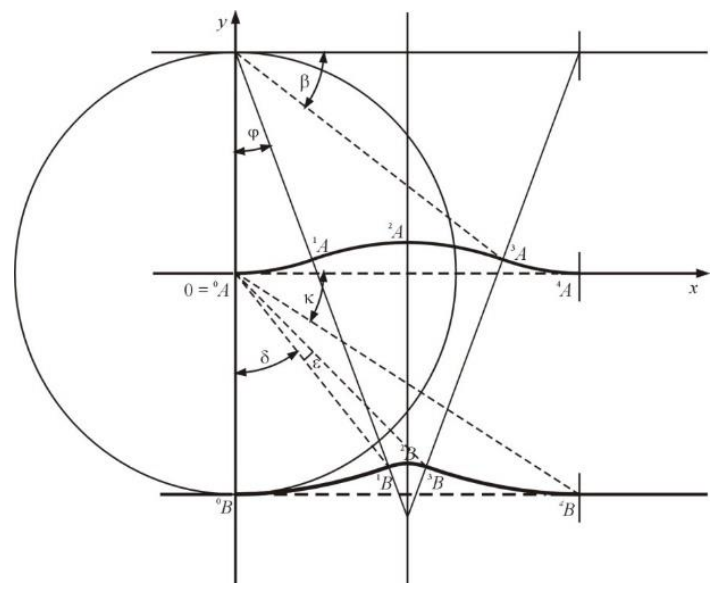

Fig. 4. Optimized speed breaker type.

Also for this model, too we first look for a mathematical expression of the road with the speed breaker. The parametric equations of road surface are as follows:

$$
\begin{aligned}
& I . k: \quad x=-r \cot t \\
& y=-r \\
& I I . k: \quad x=r\left(\sin t+\sqrt{(\sin t)^{2}+3}\right) \cos t \\
& y=r\left(\sin t+\sqrt{(\sin t)^{2}+3}\right) \sin t \quad \text { where } t \in\left[\pi ; \frac{3}{2} \pi\right] \\
& \text { III .k } \quad x=\left(\frac{l}{2} \cos t+(h-r-R) \cos t-\sqrt{\left(\frac{l}{2} \cos t+(h-r-R) \cos t\right)^{2}-2 r(R+h)-r^{2}}\right) \cos t \\
& y=\left(\frac{l}{2} \cos t+(h-r-R) \cos t-\sqrt{\left(\frac{l}{2} \cos t+(h-r-R) \cos t\right)^{2}-2 r(R+h)-r^{2}}\right) \sin t \\
& \text { where } t \in\left[\frac{3}{2} \pi+\delta ; \frac{3}{2} \pi+\delta+\varepsilon\right]
\end{aligned}
$$




$$
\begin{array}{r}
I V . k: \quad x=\left(l \cos t+r \sin t+\sqrt{(l \cos t+r \sin t)^{2}-l^{2}+3 r^{2}}\right) \cos t \\
y=\left(l \cos t+r \sin t+\sqrt{(l \cos t+r \sin t)^{2}-l^{2}+3 r^{2}}\right) \sin t \\
\text { where } t \in\left[\frac{3}{2} \pi+\delta+\varepsilon ; 2 \pi-\kappa\right]
\end{array}
$$

V.k.: $\quad x=-r \cot t$

$$
y=-r \quad \text { where } t \in[2 \pi-\kappa ; 2 \pi]
$$

We used a cubic interpolation polynomials (splines) to derive the shape of road surface with the speed breaker. We chose the construction of a natural cubic spline. Because $S^{\prime \prime}(0)=$ $S^{\prime \prime}(l)=0$ (the values of the second derivatives of the interpolated function at its endpoints), the endpoints are the points ${ }^{0} B=[0 ;-r],{ }^{4} B=[l ;-r]$, and the joining points are ${ }^{1} B=$ $\left[\frac{r l}{2 r+R} ; \frac{2 r h}{2 r+R}-r\right],{ }^{2} B=\left[\frac{l}{2} ; h-r\right]$, and ${ }^{3} B=\left[\frac{(r+R) l}{2 r+R} ; \frac{2 r h}{2 r+R}-r\right]$.

Thus, we have also created this model in an explicit form from five segments and their equations are in the form of polynomial functions of degree 3 [3]. Analogously, we proceeded to model the trajectory of a moving vehicle along the optimized speed breaker. The structure of the trajectory consists of similar parts to the speed breaker and it has a similar shape (Fig. 4). Parametric equations of vehicle's trajectory are as follows:

$$
\begin{aligned}
& \text { I. } t: \quad x=-r \cot t \\
& y=0 \\
& \text { II. } t: \quad x=r \cos t \\
& y=r \sin t+r \\
& \text { where } t \in\left[\pi ; \frac{3}{2} \pi\right] \\
& \text { III. } t: \quad x=\left(\frac{l}{2} \cos t+(h-2 r-R) \sin t-\sqrt{\left(\frac{l}{2} \cos t+(h-2 r-R) \sin t\right)^{2}-3 r^{2}-2 r R}\right) \cos t \\
& y=\left(\frac{l}{2} \cos t+(h-2 r-R) \sin t-\sqrt{\left(\frac{l}{2} \cos t+(h-2 r-R) \sin t\right)^{2}-3 r^{2}-2 r R}\right) \sin t+r \\
& \text { where } t \in\left[\frac{3}{2} \pi+\varphi ; 2 \pi-\beta\right] \\
& \text { IV.t.: } x=\left(l \cos t+\sqrt{r^{2}-l^{2}(\sin t)^{2}}\right) \cos t \\
& y=\left(l \cos t+\sqrt{r^{2}-l^{2}(\sin t)^{2}}\right) \sin t+r \quad \text { where } t \in[2 \pi-\beta ; 2 \pi-\kappa] \\
& \text { V.t: } \quad x=-r \cot t \\
& y=0 \\
& \text { where } t \in[2 \pi-\kappa ; 2 \pi]
\end{aligned}
$$

The explicit expression of the trajectory was achieved in the same way as for the optimized speed breaker, i.e., using the construction of a natural cubic spline. The spline endpoints are ${ }^{0} A=[0 ; 0],{ }^{4} A=[l ; 0]$, and the joining points are ${ }^{1} A=\left[\frac{r l}{2(2 r+R)} ; \frac{r h}{2 r+R}\right]$, ${ }^{2} A=\left[\frac{l}{2} ; h\right]$ and ${ }^{3} A=\left[\frac{l(3 r+2 R)}{2(2 r+R)} ; \frac{r h}{2 r+R}\right]$. The model of this trajectory in an explicit form also has five segments and their equations are in the form of degree 3 polynomial functions [3].

The less curvature $k$ around a given point is, the smaller the flexion value $\chi_{T_{0}}$ at this point and the greater the radius of the rolling circle (tire) $r_{T_{0}}$. Therefore, we are looking for an approximation of the road profile using a continuous curve with a suitable curvature that has the desired shape and can be expressed by a single function. The witch of Agnesi curve with the equation $y(x)=\frac{h^{3}}{h^{2}+x^{2}}$ satisfies these requirements. The basic version, however, has greater flexion $\chi_{T_{0}}$ than $1 / R$. In this case, the standard speed breaker is located below the road profile (Fig. 5) and the vehicle loses contact with the road at low speed. 


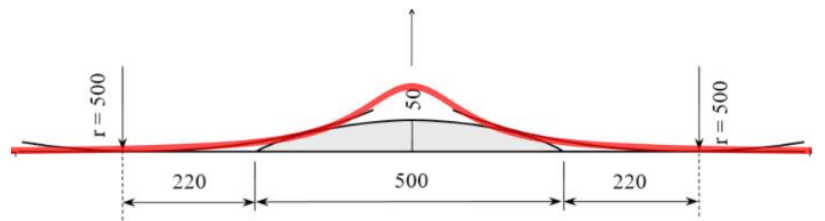

Fig. 5. The witch of Agnesi road profile

Therefore we adjust the equation of the curve to the form: $y(x)=h^{3} \cdot\left(h^{2}+k^{-1} x^{2}\right)^{-1}$. To make the graph of the function $y(x)$ coincide with the standard speed breaker profile, we place the origin of the coordinate system at the point at which the curve attains the maximum as shown in Fig. 6. Since $\chi_{T}(0)=\frac{2}{k h}$, the constant $k$ satisfies: $k \geq \frac{2 R}{h}$.

It is known to construct the witch of Agnesi curve using a circle. However, if we use an ellipse with a ratio of axes $a: b=h / 2$ instead of a circle, then we use this ellipse to construct the profile with the equation: $y(x)=h^{2} \cdot\left(h+\left(\frac{x}{2 R}\right)^{2}\right)^{-1}$. The explicit expression of the trajectory was again achieved in the same way: $y(x)=h^{2} \cdot\left(h+\left(\frac{x}{2(R+r)}\right)^{2}\right)^{-1}+r$, where $r$ is the radius of the wheel (Fig. 6).

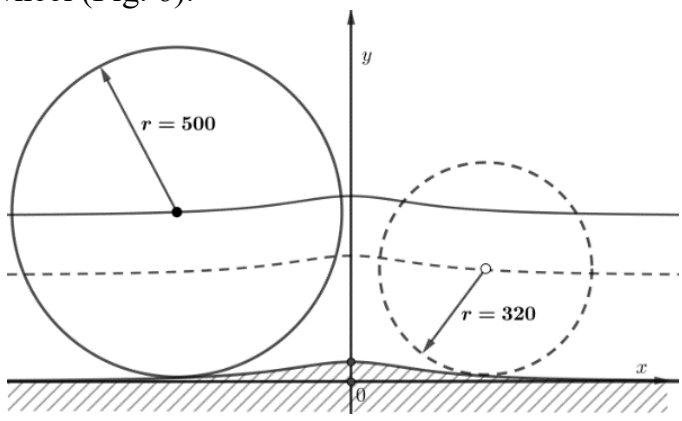

Fig. 6. Trajectories for different radii.

To simplify the model we again neglected the deformation of the tire. When modeling the profile, we required the model function to have a zero derivative at the endpoints. The modeled profile with the witch of Agnesi curve does not prevent it from having a derivative at the endpoints close to zero. The benefit of this model, however, is that it is described as a single continuous function for all real numbers.

\section{Conclusions}

Unevenness encountered on roads other than deliberately imposed speed breakers usually result from the dynamic effects of moving vehicles, the effects of weather changes, and road reconstruction. A vehicle moving on the road is often forced to adapt its speed to the conditions and shape of the road. It is therefore appropriate to deal with modeling the vehicle's trajectory on a road with uneven surface. The results obtained from numerical analysis combined with experimental analysis will serve both vehicle and road designers, road managers in terms of maintenance and ensuring durability, as well as drivers with regards to driving safety.

This paper was supported by the Grant National Agency VEGA of the Slovak Republic (grant No. $1 / 0006 / 20)$. 


\section{References}

1. J. Melcer, G. Lajčáková, I. Martinická, J. Králik, Dynamics of Transport Structures (EDIS ŽU Žilina, 374 p., 2016)

2. J. Melcer, D. Stachová, On one problem in solving vehicle-road interaction. In: Dynamics of civil engineering and transport structures and wind engineering DYN-WIND’2003, Tále, Slovak Republic, University of Žilina, 178-181 (2003)

3. D. Stachová, Elimination of singular points during a passage over a speed breaker. In: Dynamics of civil engineering and transport structures and wind engineering DYN-WIND’2011, Jasná, Slovak Republic, University of Žilina, 235-238 (2011)

4. J. Melcer, D. Martinická, Civil and Environmental Engineering, 7, 1, 13-26 (2011)

5. G. Lajčáková, I. Martinická, J. Melcer, L. Daniel, Civil and Environmental Engineering, 8, 1, 63-77 (2012)

6. L. Daniel, V. Valašková, J. Kortiš, Civil and Environmental Engineering, 10, 2, 108-112 (2014)

7. J. Melcer, D. Martinická, Civil and Environmental Engineering, 6, 1, 62-69 (2010)

8. J. Melcer, D. Martinická, Civil and Environmental Engineering, 6, 2, 128-137 (2010) 